\title{
Primeros datos de bioacumulación de metales pesados en briofitas
}

\section{First data of bioaccumulation of heavy metals in bryophytes}

\author{
Valentina Toledo Bruzual ${ }^{1}$, Diana María Hernández de Szczurek ${ }^{2}$, Caribay Urbina de \\ Navarro $^{3}$ y Francesca Creazzola de Oppeltz ${ }^{4}$
}

\begin{abstract}
Resumen
En Venezuela, existen estudios de la calidad del aire utilizando líquenes sin que aún exista un reporte con briofitas terrestres. En tal sentido, la presente investigación tiene como propósito, evaluar la capacidad de las briofitas autóctonas mediante el estudio de la bioacumulación de metales pesados, a través del monitoreo pasivo. Para el desarrollo del estudio se recolectaron distintas briofitas, en un área natural protegida llamada Topo Itagua, localizada detrás de la Universidad Católica Andrés Bello (UCAB). Se analizaron los contenidos en $\mathrm{Fe}, \mathrm{Cd}, \mathrm{Zn}, \mathrm{Cu}$ y $\mathrm{Pb}, \mathrm{con}$ espectrometría de emisión óptica de plasma acoplada inductivamente (ICP-OES) y $\mathrm{Hg}$ con analizador por fluorescencia. Los factores de enriquecimiento se calcularon a través de suelos de in situ, no impactados por actividades antropogénicas. Los resultados muestran las primeras briofitas Tortella humilis y la Tortula sp. como potenciales bioacumuladores de $\mathrm{Zn}$, así como la Pottiaceae sp. de bioacumular $\mathrm{Zn}$ y Cd. El estacionamiento de profesores, es el lugar con los Factores de Enriquecimiento más alto con Cd y Zn y podría considerarse como la zona de riesgo para la población que trabaja en la UCAB. Se sugiere continuar en sectores aledaños al Topo Itagua a fin de aumentar la densidad del muestreo para ampliar el tipo de entorno y consolidar el material de referencia del suelo UCAB.
\end{abstract}

Palabras clave: metales pesados; briofitas terrestres, bioacumulador; ICP-OES; monitoreo pasivo

\begin{abstract}
In Venezuela, there are studies of the air quality using lichens the absence of even a report with bryophytes land. In this sense, this research aims to, assess the capacity of the bryophyte native through the study of the bioaccumulation of heavy metals, through the passive monitoring. The development of the study was collected different bryophytes, in a protected area called Topo, Itagua, located behind the Universidad Católica Andrés Bello (UCAB). The contents were analyzed in $\mathrm{Fe}, \mathrm{Cd}, \mathrm{Zn}, \mathrm{Cu}$ and $\mathrm{Pb}$, with inductively coupled plasma optical emission spectrometry (ICP-OES) and Hg by fluorescence Analyzer. Enrichment factors were calculated through soils in situ, not impacted by anthropogenic activities. The results show the first bryophyte Tortella humilis and the Tortula sp. as potential accumulator of $\mathrm{Zn}$, as well as the Pottiaceae sp. of $\mathrm{Zn}$ and Cd bioacumular. The teachers parking lot, is the place with the highest enrichment with $\mathrm{Cd}$ and $\mathrm{Zn}$ factors and may be considered the danger zone for the people working at the UCAB. It is suggested to continue in the Topo Itagua adjacent sectors in order to increase sampling density to expand the type of environment and consolidate the UCAB ground reference material.
\end{abstract}

Key words: metal heavy; terrestrial bryophytes; bioaccumulator; ICP-OES; passive monitoring.

\footnotetext{
${ }^{1}$ Profesora en Geografía, Titular, Universidad Pedagógica Experimental Libertador, Instituto Pedagógico de Caracas, Departamento de Geografía, toledo.valentina@gmail.com.

${ }^{2}$ Profesora en Química, Titular, Universidad Pedagógica Experimental Libertador, Instituto Pedagógico de Caracas, Departamento de Biología y Química,dmhernandezs@gmail.com

${ }^{3}$ Lic en Química, Titular, Universidad Central de Venezuela, Facultad de Ciencia, Centro de Microscopia Electrónica, "Mitsuo Ogura", caribayurbina@gmail.com

${ }^{4}$ Profesora en Química, Titular, Universidad Pedagógica Experimental Libertador, Instituto Pedagógico de Caracas, Departamento de Biología y Química, fcreazzola@hotmail.com
} 


\section{Introducción}

En la organización espacial de las ciudades es frecuente visualizar un defecto de fondo en el modelo de crecimiento urbanístico, prueba de esto son los múltiples focos de contaminación ligados a procesos industriales incluidos en los núcleos urbanos. Un ejemplo de lo anterior lo representa claramente el sector popular de Carapita y zona industrialresidencial de Atímano, las cuales colindan con la zona industrial de La Yaguara.

Las técnicas habituales para la medición de las concentraciones de contaminantes en la atmósfera emplean métodos físicos y químicos, requiriendo a menudo prácticas sofisticadas y aparatos automáticos y semiautomáticos. Las medidas en continuo en puntos fijos (normalmente como parte de redes de control), así como las realizadas con equipos móviles permiten la valoración de los niveles y los flujos de los macro-contaminantes atmosféricos (DIGESA 2005). Estas medidas se usan para detectar concentraciones de contaminantes y verificar la efectividad de las medidas adoptadas para reducir la contaminación atmosférica.

El monitoreo biológico ofrece una medida del impacto que pueden causar las acumulaciones de los contaminantes sobre los seres vivos, informando de las posibilidades de entrada en la cadena trófica y de sus efectos antagónicos y sinérgicos. Entre las ventajas del biomonitoreo está la posibilidad de realizar mediciones de múltiples elementos (micro y macro contaminantes) y el diseño de redes con la densidad adecuada a cada tipo de entorno o estudio; mientras que las técnicas convencionales se ven limitadas por la falta de tecnología o por los costes que implicarían instalar redes similares (Ahmad et al., 2007; Nuñez, 2009).

Los líquenes tienen un importante papel como bioindicadores de lectura inmediata de la contaminación medioambiental, de los cambios climáticos y de la estabilización del suelo (Hawksworth et al., 2005). Los mismos autores señalan que se han desarrollados muchos trabajos acerca de este tema en regiones templadas, pero tan solo en algunos pocos casos las técnicas empleadas en este tipo de estudios han sido utilizados en los trópicos. Tal es el caso, de la investigación realizada por Hurtado (2010), en la cual realizó un monitoreo de tipo activo con líquenes trasplantado de la especie Parmotrema Sancti Angelli en los alrededores de la población minera de Tiara, con el objetivo de evaluar la calidad del aire en relación a fuentes fijas (Empresa Minera Loma de Níquel, C.A). Los resultados obtenidos evidencian que existe acumulación para todos los metales, a excepción del Cd y As que no fueron detectados en las muestras trasplantadas. En este mismo orden de idea, la investigación de Mendoza (2018), en Chachapoyas, la principal ciudad de Amazonas que señala la correlación entre la diversidad de líquenes y el Índice de Pureza Atmosférica Modificado (IPAM) con las variables ambientales y las especies de forofitos. Con los datos del IPAM realizó los mapas de isocontaminación, utilizando el interpolador Kriging en el programa ArcGi. Los resultados muestran la distribución de la contaminación del aire de la ciudad, principalmente por $\mathrm{SO}_{2}$ según la indicación liquénica.

Por otra parte, el uso de briofitas terrestres ha sido una herramienta empleada en numerosos estudios desde que en 1968 comenzaran a utilizarse para el control de la calidad del aire (Rühling y Tyler 2001). Su utilización como biomonitores se debe a una serie de características morfológicas, fisiológicas y ecológicas. Hay amplias evidencias que las briofitas son excelentes indicadores de la contaminación ambiental y algunos autores han sugerido usarlas como briómetros, instrumentos para medir la fototoxicidad de la contaminación ambiental (Taoda 1973). Aún más, muchas especies de briofitas han perdido su fertilidad o se han extinguido en las áreas urbanas por su gran sensibilidad a la contaminación ambiental (Le Blanck y Rao 1973).

Las briofitas debido a su simplicidad estructural carecen de una cutícula, de un sistema vascular y de un sistema radicular bien desarrollados, absorben la mayor parte de los minerales necesarios para su desarrollo por vía de la deposición atmosférica (seca y/o húmeda) y no del sustrato, reduciendo la posible influencia de los metales pesados presentes en el sustrato en el que viven (Fernández et al., 1999).

La sensibilidad de las briofitas a la contaminación crece desde las formas terrícolas (en el suelo de los bosques) a las saxícolas (en rocas y muros) y las epífitas (árboles y ramas). Según señala Matteri (2010), esta sensibilidad de las 
briofitas ha sido especialmente investigada para los gases tóxicos del aire como el dióxido de azufre $\left(\mathrm{SO}_{4}\right)$, ácido sulfúrico, $\left(\mathrm{H}_{2} \mathrm{SO}_{4}\right)$ y ozono $\left(\mathrm{O}_{3}\right)$. También son capaces de concentrar metales pesados como bioacumuladores en grandes cantidades, como zinc $(\mathrm{Zn})$, plomo $(\mathrm{Pb})$, hierro $(\mathrm{Fe})$, cobre $(\mathrm{Cu})$, níquel $(\mathrm{Ni})$ y cadmio $(\mathrm{Cd})$, entre otros (Fernández et al., 1999; Aceto 2003; Pesch y Schroeder 2006; Anze et al., 2007; Noriega 2008). En tal sentido, Cango (2015), señala que el uso de las comunidades de líquenes y briófitos como indicadores ha mostrado ser una alternativa rápida, eficiente y de bajo costo para la evaluación de la calidad del aire.

Ahora bien, cuando los biomonitores que se utilizan son aquellas especies que se encuentran presentes en los ecosistemas a evaluar, entonces se habla de biomonitoreo pasivo (Noriega, 2008). En ausencia de musgo nativo o autóctono (biomonitoreo pasivo) puede emplearse el biomonitoreo activo basado en trasplantes de musgos (técnica conocida como "moss bags" por su denominación en inglés, Tyler 1990). En los últimos años, ambas técnicas han sido empleadas con éxito en el control de la calidad del aire sobre todo en áreas urbanas e industriales (Ahmad et al., 2007). Ecólogos en el mundo, han investigado el dramático empobrecimiento de las comunidades briofíticas en los alrededor de las grandes ciudades y áreas industriales a consecuencia de la contaminación atmosférica Barkman (citado en Matteri 2010).

Los trabajos citados en los párrafos anteriores muestran la eficiencia relativa de la acumulación de metales pesados en el musgo (briofitas), normalizando el contenido corporal de la especie de musgo utilizado, frente a una especie control. También se ha determinado la eficiencia de las briofitas, considerando la concentración corporal en el musgo frente a una muestra de suelo, tomando como referencia un elemento, por ejemplo el aluminio (Al). Para el primer caso, se habla de niveles de fondo (NF) para el segundo caso, factor de enriquecimiento (FC). El estudio de la acumulación o enriquecimiento de metales se basa en técnicas de normalización geoquímica, las cuales permiten obtener información acerca de la presencia de una sustancia o elemento que ha sido transportado por lluvia, viento, etc. hasta otro medio u organismo (Del Águila et al., 2005). Garcia et al. (2016), prepresenta una alternativa a los tipos de monitoreo tradicionales (pasivo y activo), ya que propone evitar la extracción del musgo nativo de los sitios de monitoreo, en su lugar plantea el cultivo in vitro del musgo y su posterior exposición en los sitios de monitoreo.

Una amplia revisión bibliográfica realizada por Merwin y Nadkarni (2002), destaca que es notorio el uso de las briofitas como indicadores biológicos de contaminación atmosférica en las regiones tropicales (entre el Trópico de Cáncer y el Trópico de Capricornio). Recientemente, Stanković, et al. (2018), detallas los conocimientos sobre los efectos de contaminación y acumulación de metales pesados en briofitas, mecanismos de tolerancia y resistencia que presentan. En ambas investigaciones no reporta su uso en Venezuela.

Considerando la superficie de Venezuela y la gran variedad de su topografía, el número de briofitas conocidos de este país es pequeño según (Pursell 1973). Solo aparecen descripciones de especies en censos y catálogos (Ramírez y Crusco De DALL'AGLIO 1979; Moreno 1992), así como informes de especies nuevas (León 2005). Igualmente, Morales et al., (2008), realizaron un inventario brioflorístico en el área recreativa del Jardín Botánico de Caracas, registrando 29 especies de briofitas divididas en hepáticas foliosas y musgos, añadiendo 14 nuevos registros a la brioflora del Distrito Capital. En esta investigación concluyen que las briofitas reportadas tienen una amplia distribución geográfica e incluso altitudinal, así como una gran capacidad adaptativa a hábitats con modificaciones importantes. Además recomiendan utilizar especies de la brioflora como las citadas: Entodon beyrichii, Fabronia ciliaris, Fissidens angustifolius, F. reticulosus, Chryso-hypnum diminutivum, Vesicularia vesicularis, Leucomium strumosum, Entodontopsis leucostega, E. nitens, Trichostomum brachydontium y Weissia controversa, para evaluar la calidad del aire en la ciudad de Caracas, es decir usar dichas especies como indicadores de contaminación atmosférica. Posteriormente, Morales (2009) realizó un muestreo durante un año en la vertiente sur del parque Nacional el Ávila, en un perfil altitudinal, en seis zonas. Los resultados florísticos de este trabajo aportan 22 adiciones a la flora muscícola de la cordillera de la costa y cinco nuevos registros para la brioflora venezolana.

Recientemente, Delgado y León (2017), presentaron un listado de 90 especies de musgos asociados a parches de bosques de Polylepis sericea Wedd., en el estado Mérida, Venezuela. El análisis fitogeográfico muestra una 
predominancia del elemento neotropical (36\%) sobre el elemento ampliamente distribuido en el mundo (27\%). En la Sierra Nevada de Mérida, son exclusivas 39 especies y 34 en la Sierra de La Culata. Solo el 19\% de las especies es compartido por ambas sierras, indicando que existe un recambio de las especies en los parches de bosque inventarios lo que podría estar asociado con variables físicas y ambientales de cada localidad. Además, agregan las especies Drepanocladus sordidus (Amblystegiaceae), Myurella sibirica (Plagiotheciaceae) y Pleurochaete luteola (Pottiaceae) a la brioflora venezolana, así como nueve nuevos registros para la brioflora del estado Mérida.

Lo reportado en las diferentes investigaciones revisadas, sugiere que en Venezuela al parecer aún no se han realizado estudios utilizando las briofitas para determinar contaminación del aire. En este sentido, la realidad de las actividades observadas en las zonas aledañas a la Universidad Católica Andrés Bello, motiva a pensar que existe la posibilidad de identificar algunas especies de briofitas autóctonas, diferentes a las anteriormente citadas que crezcan en los suelo y rocas con gran capacidad adaptativa a hábitats con múltiples focos de contaminación (zona industrial, tránsito vehicular, etc.), que pudieran utilizarse como bioacumuladores de metales pesados de origen antropogénico. Las líneas de trabajo en Venezuela han estado orientadas hacia la utilización de líquenes para tal fin, sin que aún exista un reporte con briofitas terrestres. Por tanto, la presente investigación tiene como propósito, evaluar la capacidad de las briofitas autóctonas mediante el estudio de la bioacumulación de metales pesados, a través del monitoreo pasivo e inferir en el posible origen de los metales, así como los sitios que podrían representar un riesgo potencial para la salud. Para el desarrollo del estudio se recolectaron distintas briofitas, en un área natural protegida llamada Topo Itagua, localizada detrás de la Universidad Católica Andrés Bello (UCAB).

\section{Materiales y Métodos}

La elección del método de análisis dependerá del propósito de la investigación. Los métodos disponibles para el monitoreo de musgo han sido evaluados por (Steinnes et al. 1993; Noriega, 2008). Mencionan entre otros, el análisis por activación neutrónica instrumental (INAA), la espectrometría de emisión óptica y de masa por plasma de acoplamiento inductivo, respectivamente (ICP-OES e ICP-MS), espectrometría de absorción atómica y cromatografía de gases. Algunos métodos de análisis no son destructivos por ejemplo, la activación de neutrones y son útiles para estudios repetitivos, tales como estudios de referencia. Las técnicas destructivas incluyen espectrometría de absorción atómica y el análisis de plasma acoplado inductivamente. Para esta investigación se seleccionó el (ICP-OES), por la disponibilidad del equipo en el país. Es importante acotar que el Hg fue analizado por otro método como el de fluorescencia debido a su volatilidad.

Para evaluar las briofitas autóctonas a través de la técnica de monitoreo pasivo, se diseñó un protocolo con 5 etapas, de la siguiente forma 2.1) criterios para la selección de las estaciones de muestreo ,2.2) recolección y registro de las briofitas y muestras de suelo, 2.3) identificación y clasificación de las briofitas, por parte de un botánico, 2.4) análisis químico y 2.5) cálculo del Factor de Enriquecimiento (FE).

\subsection{Criterio para la Selección de las Estaciones de Muestreo}

El Topo Itagua en un área natural protegida de relieve montañoso, ocupa aproximadamente $127.500 \mathrm{~m}^{2}$, se encuentra localizada dentro de las coordenadas geográficas de los $10^{\circ} 27^{\prime} 49^{\prime \prime}$ y $10^{\circ} 27^{\prime} 52$ ” N; y $66^{\circ} 58^{\prime} 30^{\prime \prime}$ y $66^{\circ} 58^{\prime} 47^{\prime \prime}$ O. No se encuentra impactada por actividades antropogénicas, por lo que fue escogida para el biomonitoreo de posibles focos de contaminación, provenientes principalmente de las actividades industriales de la zona de Antímano y la Yaguara, así como también del tráfico automotor (autopista Francisco Fajardo y Avenida Teherán), que a diario afecta a la zona residencial de Juan Pablo II, Montalbán II, el sector de Antímano y el área de la UCAB.

A nivel internacional, la colección de las briofitas se puntualiza hacia un área geográfica de interés, con un mínimo de elementos que afecten localmente la metodología (Cenci 2008). La recolección se hizo preferentemente en lugares donde se observaron abundantes briofitas o mantos de éstas y lejos de la presencia de árboles, es decir en 
pequeños claros de bosques, en el suelo o sobre rocas de pequeño tamaño, con el fin de evitar la posible intervención del lavado de la cubierta vegetal (dosel) producto de las lluvias y/o los aportes de hojarascas (tejido vivo o muerto). Las estaciones de muestreo se localizaron, al menos, a $700 \mathrm{~m}$ de arterias viales principales, núcleos urbanos e industrias, y a aproximadamente $900 \mathrm{~m}$ de carreteras secundarias y edificaciones aisladas (Ver figura 1, puntos verdes enumerados).

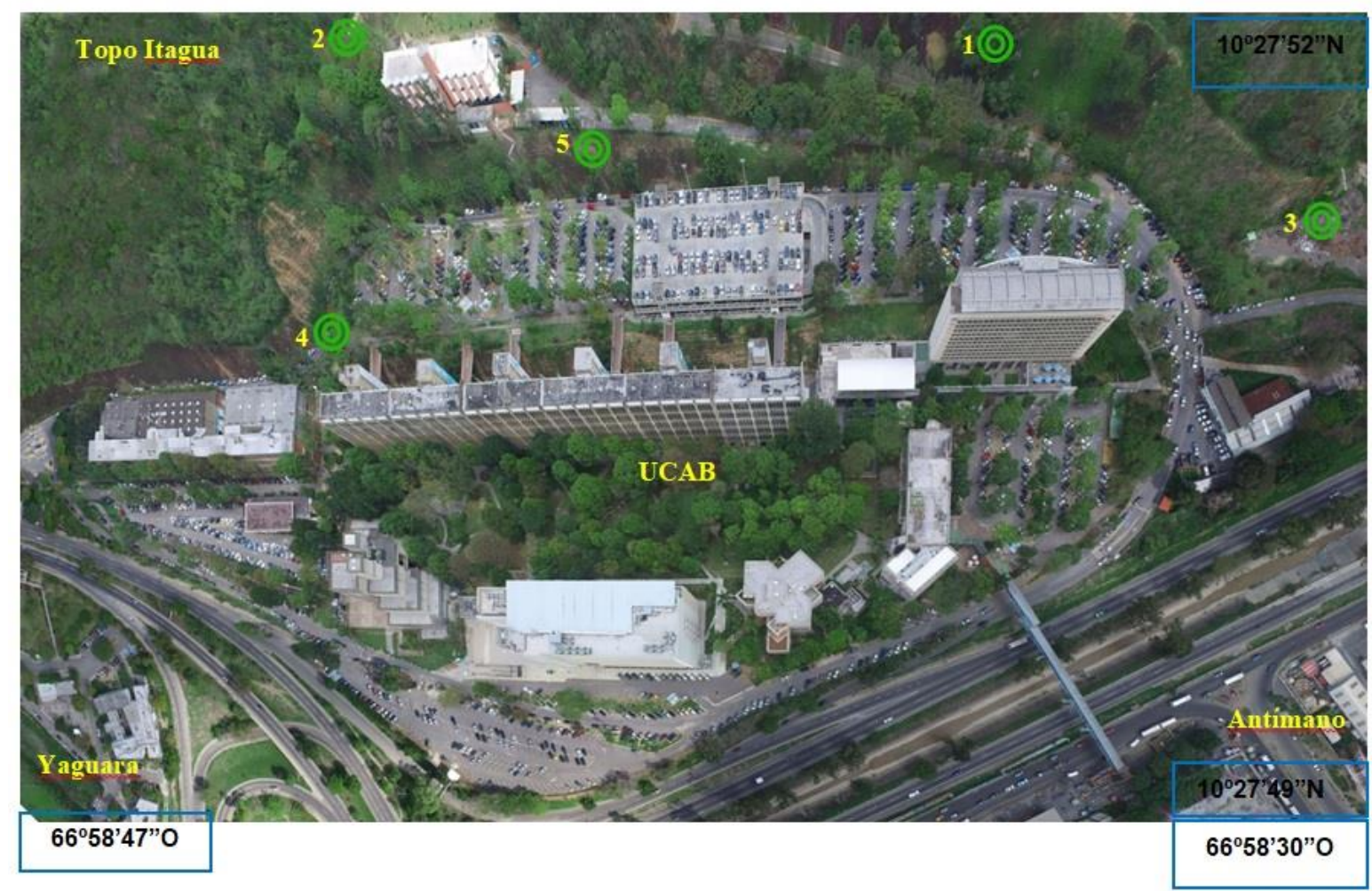

https://www.dronepixel.com.ve/wp-content/uploads/2016/05/Ucab_Panorama1-copy-1-e1515702340607.jpg

Figura 1. Localización geográfica del área de estudio y estaciones de muestreo

\subsection{Recolección y Registro de las Muestras de Briofitas y Muestras de Suelo}

En cada punto de recogida se tomó una muestra de briofitas, dentro de un área de $50 \mathrm{~cm}$ x $50 \mathrm{~cm}$. De esta manera se contó con 5 muestras para el análisis. Las características de los sitios de muestreo se especifican en la Tabla 1. Para la toma de la muestra se empleó una espátula pequeña, la muestra se limpió de hojas, espinas u otro material grueso, utilizando para ello, guantes de goma. Posteriormente, se trasladaron en bolsa transparente hermética. La recolección de las muestras de suelo se hizo in situ, en el mismo punto donde se tomaron las muestras de briofitas a una profundidad no mayor a $5 \mathrm{~cm}$ del suelo, utilizando una pala, totalizando 5 muestras de suelo. Igualmente se removió con espátula residuos de hojas, lombrices, telarañas o cualquier material grueso, se colocaron en bolsa plástica. Posteriormente, cada bolsa fue rotulada con código de muestreo, lugar de la toma y fecha. Cada muestra fue georreferenciada con el equipo portátil, etrex Summit, Garmin. Finalmente, se tomaron fotos en cada sitio de recolección y panorámica cercana. 
Tabla 1. Características de los puntos de muestra

\begin{tabular}{|c|c|c|c|c|c|}
\hline Muestra & Localidad & $\begin{array}{l}\text { Coordenadas } \\
\text { Geográficas }\end{array}$ & $\begin{array}{l}\text { Altitud } \\
\text { m.s.n.m. }\end{array}$ & Geología/ Litología & Vegetación \\
\hline $\begin{array}{l}\text { Parada 1 } \\
\text { PI }\end{array}$ & $\begin{array}{l}\text { Ladera, detrás del } \\
\text { edificio Cincuentenario, } \\
\text { lado izquierdo }\end{array}$ & $\begin{array}{l}\text { Lat: } \\
10^{\circ} 27^{\prime} 46^{\prime \prime} \mathrm{N} \\
\text { Long: } 66^{\circ} 58^{\prime} 39^{\prime \prime} \mathrm{O}\end{array}$ & 1055 & $\begin{array}{l}\text { Formación Antímano. } \\
\text { Esquistos, cuarzo micáceos } \\
\text { calcáreos y mármoles }\end{array}$ & Gramínea \\
\hline $\begin{array}{l}\text { Parada } 2 \\
\text { CB }\end{array}$ & $\begin{array}{l}\text { Ladera, campo de } \\
\text { Baseball }\end{array}$ & $\begin{array}{l}\text { Lat: } \\
10^{\circ} 27^{\prime} 41^{\prime \prime} \mathrm{N} \\
\text { Long: } \\
68^{\circ} 58^{\prime} 36^{\prime \prime} \mathrm{O}\end{array}$ & 999 & $\begin{array}{l}\text { Formación Antímano. } \\
\text { Esquistos, cuarzo micáceos } \\
\text { calcáreos y mármoles }\end{array}$ & Arbustiva \\
\hline $\begin{array}{l}\text { Parada } 3 \\
\text { PD }\end{array}$ & $\begin{array}{l}\text { Estacionamiento } \\
\text { superior, lado derecho }\end{array}$ & $\begin{array}{l}\text { Lat: } \\
10^{\circ} 27^{\prime} 46^{\prime \prime} \mathrm{N} \\
\text { Long: } \\
66^{\circ} 58^{\prime} 47^{\prime \prime} \mathrm{O}\end{array}$ & 1007 & $\begin{array}{l}\text { Formación Antímano. } \\
\text { Esquistos, cuarzo micáceos } \\
\text { calcáreos y mármoles }\end{array}$ & Arbustiva \\
\hline $\begin{array}{l}\text { Parada } 4 \\
\text { EI }\end{array}$ & $\begin{array}{l}\text { Entrada principal, detrás } \\
\text { de Ingeniería }\end{array}$ & $\begin{array}{l}\text { Lat: } \\
10^{\circ} 27^{\prime} 52^{\prime \prime} \mathrm{N} \\
\text { Long: } \\
66^{\circ} 58^{\prime} 30^{\prime \prime} \mathrm{O}\end{array}$ & 939 & $\begin{array}{l}\text { Formación Antímano. } \\
\text { Esquistos, cuarzo micáceos } \\
\text { calcáreos y mármoles }\end{array}$ & Gramínea \\
\hline $\begin{array}{l}\text { Parada } 5 \\
\text { EP }\end{array}$ & $\begin{array}{l}\text { Estacionamiento } \\
\text { profesores }\end{array}$ & $\begin{array}{l}\text { Lat: } \\
10^{\circ} 27^{\prime} 49^{\prime \prime} \mathrm{N} \\
\text { Long: } \\
66^{\circ} 58^{\prime} 31^{\prime \prime} \mathrm{O}\end{array}$ & 954 & $\begin{array}{l}\text { Formación Antímano. } \\
\text { Esquistos, cuarzo micáceos } \\
\text { calcáreos y mármoles }\end{array}$ & Arbustiva \\
\hline
\end{tabular}

\subsection{Identificación y Clasificación de las Especies de Briofitas}

Se colectó una submuestra para la identificación de la especie en cada punto de muestreo, éstas se colocaron en cápsulas Petri de polietileno previamente etiquetada.

\subsection{Análisis Químico}

El pre-tratamiento de las muestras de briofitas y las muestras de suelo, se hizo de acuerdo al procedimiento señalado por Cenci (2008), con una sola modificación, la molienda de las briofitas y de las muestras de suelo, se realizó en un mortero de acero inoxidable.

Se tomaron porciones de $1,0 \mathrm{~g}$ de polvo de briofitas y de $1,0 \mathrm{~g}$ polvo de suelos. Se añadió $15 \mathrm{~mL}$ de $\mathrm{HNO}_{3}$ para una digestión ácida por 48 horas para todas las muestras. Se reducen hasta $2,5 \mathrm{~mL}$ por evaporación con calor y se lleva a volumen con agua destilada en matrax de $100 \mathrm{~mL}$. Se filtró con papel filtro banda negra. Se procedió a cuantificar el contenido de $\mathrm{Fe}, \mathrm{Cu}, \mathrm{Pb}, \mathrm{Cd}$ y $\mathrm{Al}$ por espectrometría de emisión con plasma acoplado inductivamente (ICP-OES), marca Thermo Jarrel ASH, modelo Iris HR. Para el análisis de Hg, se utilizó un analizador de mercurio, marca Analytikjema, modelo Mercur con fluorescencia. Se efectuó cada análisis por triplicado en la Escuela de Química, Laboratorio de Analítica de la UCV (Universidad Central de Venezuela).

El criterio de selección para estudiar los seis metales pesados ( $\mathrm{Fe}, \mathrm{Cu}, \mathrm{Pb}, \mathrm{Hg}, \mathrm{Cd}$ y $\mathrm{Zn}$ ) se basó en la revisión bibliográfica, en la cual los resultados de las diferentes investigaciones sobre las concentraciones de diversos contaminantes a nivel local, hacen hincapié en la presencia de éstos metales en áreas geográficas de interés, con un mínimo de factores que afectan localmente la metodología. 


\subsection{Cálculo del Factor de Enriquecimiento (FE)}

La recolección de muestras de suelo superficiales de suelo/substrato, se realizó, con la finalidad de evaluar los "efectos de suelo" a través del factor de enriquecimiento (FE). Es un factor usado frecuentemente en el monitoreo pasivo de la calidad del aire cuando se emplea musgo o líquenes. Este factor permite comparar la concentración individual del elemento de interés (briofitas) con el elemento conservado o de referencia del suelo, de tal manera, que la estimación del FE sea una medida para inferir el origen, antropogénico o natural de los contaminantes atmosféricos (Dubey et al., 2012; Munir et al., 2012).Una vez obtenida la concentración de metales en las muestras de briofitas y suelo in situ, se aplicó la ecuación:

$$
\begin{aligned}
& \left.\begin{array}{l}
{[\mathrm{Mm}] \text { briofita }} \\
{[\mathrm{Xm}] \text { briofita }}
\end{array}\right|_{[\mathrm{Mo}] \text { suelo }} \\
& \text { donde: } \mathrm{Mm}=\text { Concentración del metal " } \mathrm{x} \text { " en la muestra } \\
& \mathrm{Xm}=\text { Concentración del elemento conservativo de referencia }(\mathrm{Al}) \text { en la muestra de briofita } \\
& \mathrm{Mo}=\text { Concentración de metal " } \mathrm{x} \text { " en suelo in situ de la (UCAB) } \\
& \mathrm{Xo}=\text { Concentración del elemento conservativo de referencia (Al) en suelo in situ (UCAB) }
\end{aligned}
$$

El Aluminio se tomó como elemento conservativo de referencia, puesto que las concentraciones en el suelo se encuentran en el orden de $1 \%$ y difícilmente pueden ser influenciadas por fuentes antrogénicas y/o de origen natural (Ford y Hasselbach 2001; Cenci 2008).

Para evaluar los niveles de enriquecimiento diversos autores han propuesto varios criterios de comparación con el objeto de establecer si un material está siendo alterado (enriquecido) y en qué grado por actividades naturales y/o antropogénicas (Munir et al. 2012). Con base en lo anterior, se propone el criterio mostrado en la Tabla 2, de Lawson y Winchester (1979) para establecer el grado de enriquecimiento que pudieran tener las briofitas en el Topo Itagua.

Tabla 2. Clases de factores de enriquecimiento de un metal pesado

\begin{tabular}{ccl}
\hline Categoría & FE & \multicolumn{1}{c}{ Interpretación } \\
\hline$(\mathrm{N})$ & $1-10$ & Abundancia no significativa, de la roca madre \\
$(\mathrm{M})$ & $>10-500$ & $\begin{array}{l}\text { Moderadamente enriquecida e indica otra fuente de } \\
\text { enriquecimiento adicional a la roca madre }\end{array}$ \\
(A) & $>500$ & $\begin{array}{l}\text { Indica un alto enriquecimiento y muestra que existe } \\
\text { una grave contaminación de origen antropogénica. }\end{array}$ \\
\hline
\end{tabular}

\section{Discusión de resultados}

En las laderas del Topo Itagua las briofitas son visibles sobre la superficie del suelo formando mantos de cobertura aislados. En la recolección, la Pottiaceae fue la familia dominante; Frahm, Zander y Gradstein (citado en Morales et al., 2008) indican que este taxón es típico de hábitats abiertos, dado a que presenta una serie de características como el hábito acrocárpico y forma de vida cespitosa. La gran mayoría de estas briofitas acrocárpicas suelen encontrarse en ambientes terrícolas (en el suelo de los bosques) y/o saxícolas (en rocas). En ciertos casos, resultó imposible realizar la descripción hasta especie, porque algunas briofitas carecían en el momento del muestreo, de los elementos 
morfológicos completos que permitieran su clasificación. Por lo tanto, en algunas briofitas se logró identificar la familia, en otras el género y en otras hasta la especie (Tabla 3).

Tabla 3. Descripción de las distintas briofitas por familia/género/especie

\section{Parada/Especimen Descripción}

\section{1 y 3. POTTIACEAE}

Tortella humilis (Hedw.)Jenn.
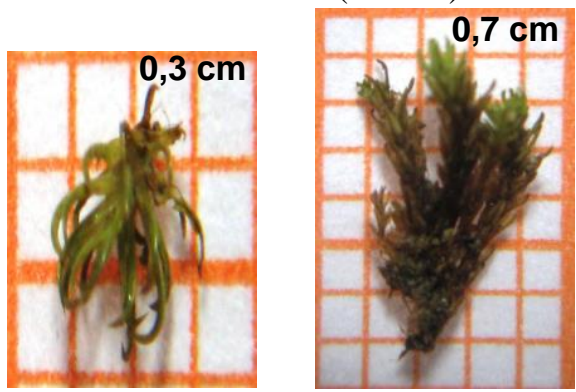

\section{POTTIACEAE}

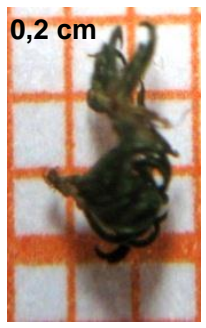

4. POTTIACEAE

Género:Tortula sp

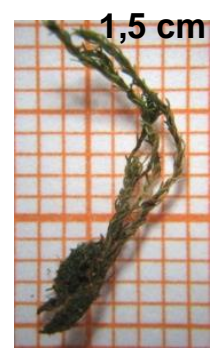

5. POTTIACEAE

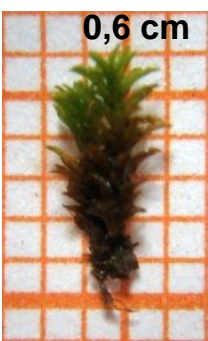

Musgo acrocárpico, de unos $0.5 \mathrm{~cm}$ de alto, hojas ligeramente retorcidas, en tres o más hileras. Costa simple percurrente; hoja acuminada y apiculada, borde entero con el margen en la parte superior recurvado. Células de la lámina foliar: la inferior rectangulares, hialinas y lisas y las superiores globodas, pequeñas y verdosas; papilosas. Apículo hialino. Esporofito con seta larga, marrón rojiza cuando adulta; cápsula cilíndrica, muy delgada, erecta. Caliptra cuculada; peristoma en una fase muy juvenil; sin embargo, se deduce que tiene dientes muy alargados. Aún no hay esporas.

Pottiaceae sin esporófito (indeterminada).

Pottiaceae sin esporófito (indeterminada).
Pottiaceae sin determinar por carencia de elementos. Musgo acrocárpico, de menos de $0.5 \mathrm{~cm}$ de alto, formando una especie de alfombra sobre el suelo; hojas linsares, encorvadas, en tres o más hileras. Apice acuminado, células foliares superiores, sapilosas. Esporofito: seta larga, lisa, verdosa; cápsula erecta, forma elipsoidal; no se observa caliptra, ni opérculo, ni peristoma. Esporas presentes. Nota: algas filamentosas, verdes y cianobacterias (Nostoc $s p$ ) presentes.

La Tabla 4, presenta las diferentes concentraciones de metales pesados en las diferentes briofitas terrestres recolectadas en el Topo Itagua. Los elementos que se encuentran en mayor abundancia en la litología Al y Fe, son 
los que aparecen con niveles más altos en las muestras vegetales. Estos datos se corresponden bastante bien con los obtenidos para la riqueza metálica en distintos tipos de rocas y en forma específica con las calizas y esquistos, las cuales son dominantes en el área de estudio (Tabla 5). Comparando los niveles dados para la litología, el Cd presenta niveles más altos en la muestra vegetal respecto a las calizas y esquistos, mientras que el $\mathrm{Zn}$, aparece con niveles más bajos en las briofitas, respecto a estos dos tipos de rocas.

Tabla 4. Análisis químicos de los contenidos corporales de las muestras de briofitas por icp/oes.

\begin{tabular}{clccccccc}
\hline Parada & $\begin{array}{l}\text { Familia/ } \\
\text { Especie }\end{array}$ & Al & Fe & $\begin{array}{c}\text { ppm } \\
\mathbf{C d}\end{array}$ & $\mathbf{Z n}$ & $\mathbf{H g}$ & $\mathbf{P b}$ & $\mathbf{C u}$ \\
\hline 1 PD & Tortella humilis & 3129 & 5610 & 1,55 & $8,0 \times 10^{-3}$ & ND & ND & ND \\
2 CB & Pottiaceae & 3393 & 5540 & 3,63 & ND & ND & ND & ND \\
3 PI & Tortella humilis & 5664 & 4780 & 0,31 & ND & ND & ND & ND \\
& & & & & & & & \\
4 EI & Tortula sp. & 2754 & 3340 & 1,45 & $9,3 \times 10^{-3}$ & ND & ND & ND \\
& & & & & & & & \\
5 EP & Pottiaceae & 519 & 1660 & 1,36 & $8,2 \times 10^{-3}$ & ND & ND & ND \\
\hline
\end{tabular}

Nota: $\mathrm{ND}=$ No detectado, menor del límite de cuantificación para cada elemento

Tabla 5. Riqueza metálica en distintos tipos de rocas (ppm)

\begin{tabular}{ccccccc}
\hline Metales & Calizas & a & Areniscas $^{\mathbf{a}}$ & Granitos $^{\mathbf{b}}$ & $\begin{array}{c}\text { Rocas }_{\text {Esquistos }}{ }^{\mathbf{b}} \\
\text { Gneises }^{\mathbf{b}}\end{array}$ & Básicas $^{\mathbf{b}}$ \\
\hline $\mathbf{A l}$ & 7000 & 43000 & & & & \\
$\mathbf{A s}$ & 1 & 1 & & & & \\
$\mathbf{C d}$ & $\mathbf{0 . 0 3}$ & 0.05 & & 0.2 & & \\
$\mathbf{C o}$ & 0.1 & 0.3 & 4 & $\mathbf{1 9}$ & 13 & 150 \\
$\mathbf{C r}$ & 11 & 35 & 12 & 90 & 76 & \\
$\mathbf{C u}$ & 5.1 & 30 & 13 & 45 & 23 & 10 \\
$\mathbf{F e}$ & 17000 & 29000 & 20000 & 48000 & 33000 & \\
$\mathbf{H g}$ & 0.16 & 0.29 & & & & \\
$\mathbf{M n}$ & 620 & 460 & 325 & 850 & 600 & \\
$\mathbf{N i}$ & 7 & 9 & 7 & 68 & 26 & 2000 \\
$\mathbf{P b}$ & 5.7 & 10 & 32 & 22 & 16 & 0.1 \\
$\mathbf{Z n}$ & $\mathbf{2 0}$ & 39 & 50 & $\mathbf{9 5}$ & 65 & 50 \\
\hline
\end{tabular}

${ }^{a}$ Salomos y Forstner (1984) ${ }^{\mathrm{b} W e d e p o h l(1991) ~ C i t a d o ~ e n ~ F e r n a ́ n d e z ~ e t ~ a l . ~(1997), 49, p .10 ~}$

Este hecho, implica que los rangos de concentración natural de los metales en los suelos pueden ser amplios y están condicionados, básicamente, por el tipo de roca madre y el grado de meteorización de la misma. Estos factores dependen, en gran medida, de la zona de estudio y, por lo tanto, existe una importante variabilidad espacial de la concentración de éstos en el suelo.

Además, la concentración natural de metales pesados puede verse modificada, por diversas actividades humanas, entre las que destacan, la actividad industrial, la producción energética, el tráfico automotor, fundiciones, etc. 
La composición química de la roca madre y los procesos de meteorización condicionan, de forma natural, la concentración de diferentes metales pesados en los suelos (Tiller 1989). La Tabla 6, muestra posible orígenes de diferentes contaminantes que pudieran asociarse con el aumento en las concentraciones naturales en el suelo.

Por consiguiente, una vez analizadas las muestras de briofitas, los datos brutos no aportan información sobre el grado de enriquecimiento que éstas presentan frente a los valores naturales (normales o de referencia). Por tanto, el problema que se plantea es saber si los resultados obtenidos son mayores que los valores naturales. A partir del análisis de un conjunto de muestras de suelo recogidas en el mismo lugar donde se recolectaron las briofitas, fue posible establecer asociaciones de la concentración de metales pesados en las briofitas con fuentes naturales o antrópicas, a través del cálculo del Factor de Enriquecimiento (FE). Los resultados se muestran en la (Tabla 7).

Tabla 6. Posibles orígenes y elementos marcadores de contaminación

\section{Posible origen Elementos marcadores}

$\begin{array}{ll}\text { Polvo de suelo } & \mathrm{Al}, \mathrm{Fe} \\ \text { Combustión carbón } & \mathrm{Al}, \mathrm{Fe}, \mathrm{S} \\ \text { Motor de Vehículos } & \mathrm{Al}, \mathrm{S}, \mathrm{Fe}, \mathrm{Pb}, \mathrm{S}, \mathrm{Zn} \\ \text { Construcción/cemento } & \mathrm{Fe}, \mathrm{S}, \mathrm{Cd}, \mathrm{Zn}, \mathrm{Pb} \\ \text { Hierro/Fundidora Acero } & \mathrm{Fe} \\ \text { Desechos de Incineradores } & \mathrm{Zn} \\ \text { Industrias } & \mathrm{Cu}, \mathrm{Pb} \\ \text { Base Metal de Acero } & \mathrm{Cd}, \mathrm{Zn} \\ \text { Incineradores de Hospitales } & \mathrm{Cd}, \mathrm{Zn}\end{array}$

Nota. Cuadro elaborado con la información de Ahmad et al. (2007).

Proc. Pakistan Acad. Sci. 44(3),205.

Tabla 7. Factor de enriquecimiento de tres elementos en briofitas utilizando el suelo como referencia $(\mathrm{n}=5)$

\begin{tabular}{|c|c|c|c|c|}
\hline \multirow[t]{2}{*}{ Parada } & $\begin{array}{c}\text { Familia/ } \\
\text { Especie o Género }\end{array}$ & \multicolumn{3}{|c|}{$\begin{array}{c}\text { Factor de Enriquecimiento } \\
\text { (FE) }\end{array}$} \\
\hline & & $\mathbf{Z n}$ & Cd & $\mathbf{F e}$ \\
\hline $1 \mathrm{PD}$ & Tortella humilis & 2,08 & 0,67 & 0,66 \\
\hline $2 \mathrm{CB}$ & Pottiaceae & - & 0,87 & 0,46 \\
\hline 3 PI & Tortella humilis & - & 0,04 & 0,15 \\
\hline $4 \mathrm{EI}$ & Tortula & 0,85 & 0,27 & 0,15 \\
\hline $5 \mathrm{EP}$ & Pottiaceae & 15,70 & 126,30 & 0,47 \\
\hline
\end{tabular}

Nota: Promontorio Izquierdo (PI), Campo de Baseball (CB), Promontorio Derecho (PD), Edificio Ingeniería (EI), Estacionamiento de profesores (EP). FE (Relación del Al en el musgo vs. material parental del suelo local).

El factor de enriquecimiento por metales pesados en las briofitas, varía en los distintos lugares donde fueron tomadas las muestras y por espécimen. La importancia de una hipotética contaminación depende del mayor o menor FE.

El promontorio Derecho del Topo Itagua (Parada 1, PD), sobre el cual crece la Tortella humilis, a una altitud $\geq 1000$ m.s.n.m aproximadamente, se encuentra fuera del alcance de los edificios de la Universidad, es decir, en dirección sureste desde donde se visualiza la zona de la Yaguara, la autopista Francisco Fajardo y la Av Teherán a una distancia de 1950 m, 975 m y 800 m respectivamente (Figura 2). Este sector presentó un FE de 2,0 con Zn es decir, un valor por debajo de 10, por lo que se deduce que su presencia en el suelo/briofita es natural, proveniente de la roca madre (categoría $\mathrm{N}$ ). 


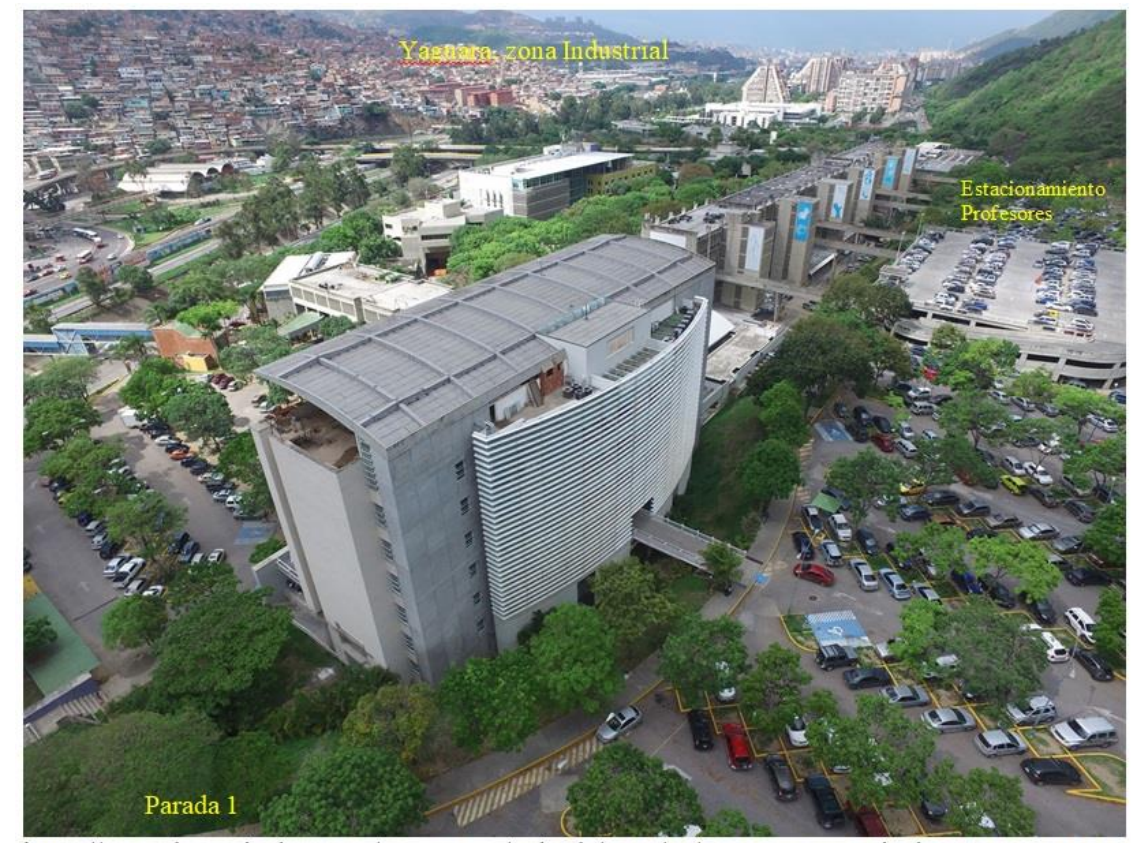

https://www.dronepixel.com.ve/wp-content/uploads/2016/05/UCAB_Drone-Pixel4-e1515702478519.jpg

Figura 2. Promontorio Derecho del Topo Itagua (Parada 1)

En cuanto a el promontorio izquierdo del Topo Itagua (Parada 3, PI), situado al suroeste, frente a los sectores de Carapita y zona industrial-residencial de Antímano, (Figura 3), se encuentra la otra Tortella humilis a la misma altura mencionada, pero en este lugar, no se detectó enriquecimiento con $\mathrm{Zn}$, a pesar de la cercanía con la autopista Francisco Fajardo, la cual se encuentra a $250 \mathrm{~m}$ con respecto al promontorio. Posiblemente, este tipo de especie es capaz de bioacumular $\mathrm{Zn}$, en bajas concentraciones cuando el foco de contaminación se encuentra a una menor distancia, el estacionamiento de profesores se localiza a 97 m de separación de la Tortella humilis en la Parada 1.

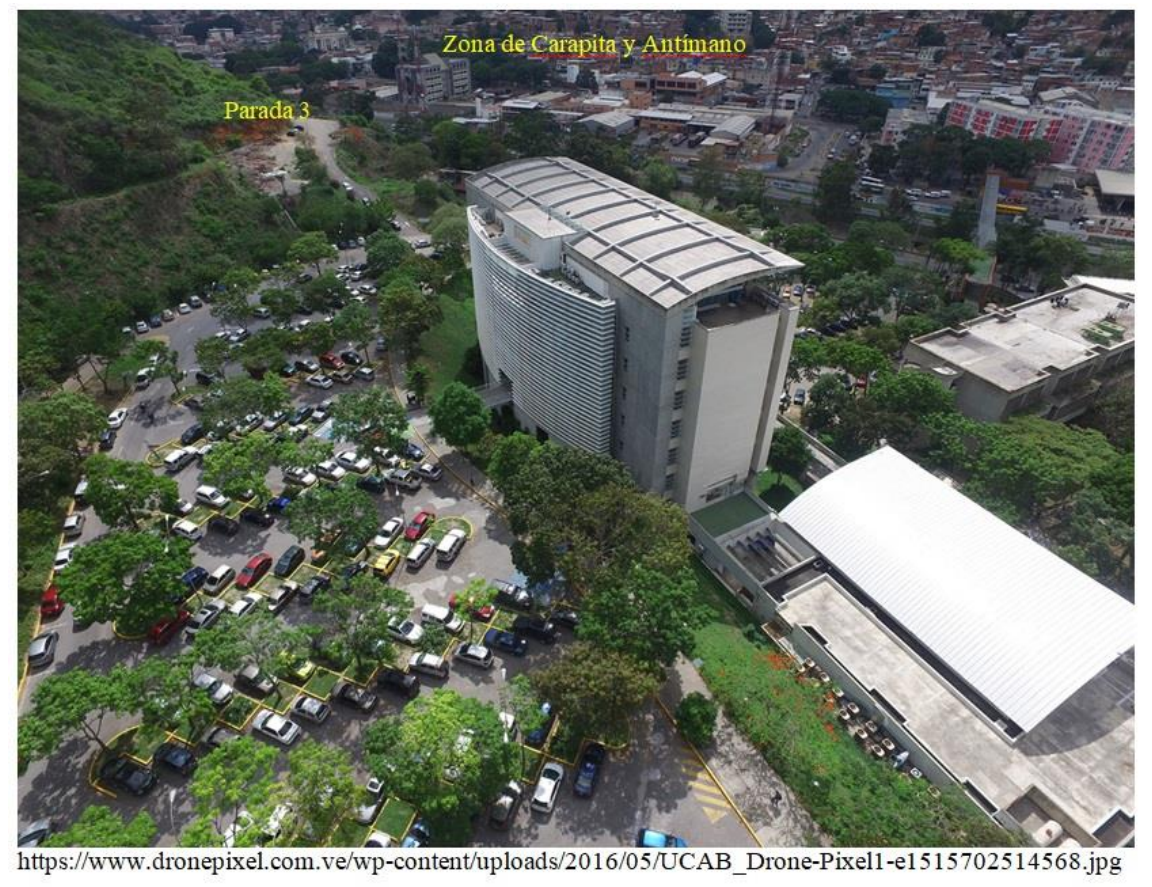

Figura 3. Promontorio izquierdo del Topo Itagua (Parada 3) 
En la (Parada 4) detrás del edificio de ingeniería, donde crece la Tortula sp., presentó un FE 0,85 con Zn, es decir, un valor por debajo de 10, por lo que se deduce que su presencia en el suelo/briofita es natural, es decir, por la meteorización de la roca madre, (categoría $\mathrm{N}$ ).

La Pottiaceae que se desarrolla en el estacionamiento de los profesores (Parada 5, EP), presenta un FE de 15,7 con $\mathrm{Zn}$, valor que es siete veces mayor al FE que presentó el promontorio derecho (Parada 1, PD) del Topo Itagua. Este valor superior a 10 indica que se trata de un metal moderadamente enriquecido en el suelo/briofita por fuentes antropogénicas, (categoría M) de acuerdo con Lawson y Winchester (1979). Pérez (1984), explica que las altas concentraciones de $\mathrm{Zn}$ obtenidas en las muestras cercanas al tránsito automovilístico posiblemente sean consecuencia del mismo tránsito y, en concreto, de las pastillas de frenado de los automóviles y al desgaste de neumáticos. Quizás sea lo que está ocurriendo en este sector de la UCAB.

En cuanto al Cd, Gerdol et al., (2000) señalan que es un elemento típico de diferentes fuentes de origen industrial y combustión de gasolina. Esta descripción, coincide con lo observado en el estacionamiento de los profesores, donde hay un gran número de vehículos que se desplaza diariamente, siendo el sitio que presenta el mayor valor de enriquecimiento de 126,3 (categoría M) por lo que indica que se trata de una metal moderadamente enriquecido en el suelo/briofita por fuentes antropogénicas. Al respecto, Aboal et al. (2010), señalan que las briofitas recogen Cd, en niveles sorprendentemente correlacionados con los emitidos. En el resto de las paradas, todas presentaron un FE por debajo de 10, por lo que se deduce que su presencia del $\mathrm{Cd}$ en el suelo/briofita se debe a la roca madre, (categoría N).

En todos los lugares muestreados, el FE para el Fe fue inferior a 10 (categoría N), lo que indica su presencia de origen geológico, es decir, natural, por meteorización de la roca. Sin embargo, el elemento Fe es suministrado principalmente a la atmósfera por el polvo y partículas del suelo transportado por viento aunque parte de las emisiones de Fe derivan de fuentes antropogénicas, especialmente por las industrias del hierro y el acero. La llegada de los vientos SE, los cuales son los predominantes en el área de estudio, probablemente sean en parte responsables del transporte de elementos desde los distintas focos de contaminación (Yaguara, autopista Francisco Fajardo, Av. Teherán y la zona de Antímano) hacia la UCAB. Discutir otros elementos climatológicos que pudieran estar influyendo en la deposición de elementos contaminantes es difícil, debido a la ausencia de estaciones climatológicas tanto en el sector de estudio como en zonas vecinas. En todo caso, el área de estudio se encuentra en la zona de referencia, que exhibe una mínima densidad de emisiones de naturaleza móvil. Esta área está poco poblada y alejada de fuentes antrópicas tanto móviles como fijas.

Posiblemente, las diferencias en la incorporación de metales pesados por las briofitas entre los distintos sitios, dependan de la disponibilidad de metales presentes a diferencias en afinidades de adsorción y/o que la deposición excede la disponibilidad de sitios de intercambio (Couto et al.2004). También es viable que las características morfológicas entre ellas y la forma de crecimiento, facilita atrapar las partículas de material suspendido, como por ejemplo, la Tortella humilis presenta una forma de crecimiento cespitosa, esto hace que presente una cubierta densa

y continua lo que naturalmente facilita atrapar mayor cantidad de partículas de polvo atmosférico, mientras que la Tortula sp. su forma de crecimiento es espigada o rastrera quedando menos expuesta al viento y por ende a la captación de partículas.

\section{Conclusiones}

Los resultados muestran las primeras briofitas Tortella humilis y la Tortula sp. como potenciales bioacumuladores de Zn, así como la Pottiaceae sp. de bioacumular Zn y Cd en el sector Topo Itagua.

El estacionamiento de profesores, es el lugar con los Factores de Enriquecimiento más alto con Cd y Zn y podría considerarse como la zona de riesgo para la población que trabaja en la UCAB. 
Se sugiere continuar en sectores aledaños al Topo Itagua a fin de aumentar la densidad del muestreo para ampliar el tipo de entorno y consolidar el material de referencia del suelo UCAB.

Hasta el cierre de la presente investigación, no se ha evidenciado estudios previos relacionados con briofitas como bioacumuladores de metales pesados en Venezuela.

\section{Agradecimientos}

Al Botánico Efrain Moreno por la identificación taxonómica de las especies de briofitas. (Laboratorio de Biología Vegetal, Departamento de Biología y Química, Instituto Pedagógico de Caracas, Universidad Pedagógica Experimental Libertador). Igualmente, a la Subdirección de Investigación y Postgrado de la Universidad Pedagógica Experimental Libertador, Instituto Pedagógico de Caracas, por el apoyo financiero para este proyecto, registrado con el N 11-183, en el Centro de Investigación en Ciencias Naturales “Manuel Ángel González Sponga” (CICNAT).

\section{Referencias bibliografias}

Aboal, J.R., Fernández, J.A., Boquete, T. y Carballeira, A. 2010. Is it possible to estimate atmospheric deposition of heavy metals by analysis of terrestrial mosses? Science of The Total Environment. 408:6291-6297.

Aceto, M., Abollino, A., Conca, R., Malandrino, M., Mentasti, E y Sarzanini, C. 2003. The use mosses as environmental mental pollution indicators. Chemosphere. 50:333-342.

Ahmad, S., Daud, M. y Qureshi, I. 2007. Use on biomonitors to assess the atmospheric chances. Proc. Pakistan. Acad. Sci. 44:201-219.

Anze, R., Franken, M., Zaballa, M., Pinto, M., Zeballos, G., Cuadros, M.A., Canseco, A., De la Rocha, A., Estellano, V y Del Granado, S. 2007. Bioindicadores en la detección de la contaminación atmosférica en Bolivia. Disponible en: http://revistavirtual.redesma.org/vol1/artículo3.htm [12 de abril de 2010].

Cango, G. 2015. Briofitos y líquenes epìfitos como organismos bioindicadores de la calidad del aire de la ciudad de Loja. Tesis de Ingeniero en gestión ambiental, Universidad Técnica Particular de Loja. Loja, Ecuador.

Cenci, R. 2008. Guidelines for the use of native mosses, transplanted mosses and soils in assessing organic and inorganic contaminant fallout. Disponible en: http://eusoils.jrc.ec.europa.eu/esdb_archive/eurosoils_docs/Other/EUR2329EN. [6 de marzo de 2011].

Couto, J., Fernández, J., Aboal, J., Carballeira A. 2004.Active biomonitoring of element uptake with terrestrial mosses: a comparison of bulk and dry deposition. Science of the Total Environment 324: 211-222.

Del Águila, P., Lugo J,y Vaca, R. 2005. Determinación de factores de enriquecimiento y geoacumulación de Cd, Cr, $\mathrm{Cu}, \mathrm{Ni}, \mathrm{Pb}$ y $\mathrm{Zn}$ en suelos de cuenca alta del río Lerma. Ciencia Ergo Sum 12:155-161.

Delgado, J., y León, Y. 2017. Musgos (Bryophyta) de bosques de Polylepis sericea (Rosaceae) del estado Mérida (Venezuela). Boletín de la Sociedad Argentina de Botánica. 52(2): 295-313.

DIGESA. 2005. Protocolo de monitoreo de la calidad del aire y gestión de los datos. Disponible en: http://www.digesa.minsa.gob.pe/DEPA/informes_aire.pdf. [01 de mayo de 2011].

Dubey, B., Asim, P., y Gurdee, S. 2012. Trace metal composition of airborne particulate matter in the coal mining and non-mining areas of Dhanbad Region, Jharkhand, India. Atmospheric Pollution Research. 3:238-246. 
Fernández, J., Puche, F., Gimeno, C. y Carballeira, A. 1999. Primeros datos sobre el biocontrol de la deposición atmosférica de metales pesados en las provincias de Valencia, Castellón y Teruel mediante musgos terrestres. Ecología.13: 83-91.

Ford, J., y Hasselbach, L. 2001. Heavy metals in mosses and soils on six transects along the Red Dog Mine Haul Road Alaska. NPS/AR/NRTR. Disponible en: http://www.dec.state.ak.us/spar/csp/docs/reddog/re. [19 de abril de 2011].

García, M., Gómez, A., Avonce, N., Zarazúa, G. y Barrera, C. 2016. Musgos cultivados, indicadores ambientales de contaminación atmosférica. Revista Mexicana de Agroecosistemas Vol. 3 (Suplemento 3):15-17.

Gerdol, R., Bragazza, L., Marchesini, R., Alber, R,. Bonetti, L., Lorenzoni, G., Achilli, M., Buffoni, A., De Marco, N., Franchi, M., Pison, S., Giaquinta, S., Palmieri, F. y Spezzano, P. 2000. Monitoring of heavy metal deposition in northern Italy by moss analysis. Environmental Pollution. 108:201-208.

Hasselbach, L., Ver Hoef, J., Ford, J., Neitlich, P., Crecelius, E., Berryman, S., Wolk, B y Bohle, T. 2005. Spatial patterns of cadmium and lead deposition on and adjacent to National Park Service lands in the vicinity of Red Dog Mine, Alaska. Science of the Total Environment. 348:211-230.

Hawksworth, D., Iturriaga, T y Crespo, A. 2005. Líquenes como bioindicadores inmediatos de contaminación y cambios medio-ambientales en los trópicos. Rev.Iberoam Micol,22:71-82.

Hurtado, W. 2010. Evaluación de la calidad del aire en los alrededores de la población minera de Tiara empleando líquenes como biomonitores de metales. Tesis de Licenciatura. Departamento de Geoquímica. Universidad Central de Venezuela. Caracas, 147p.

Lawson, D. y Winchester, J. 1979. A Standar crustal aerosols as a Reference for Elemental Enrichment Factors. Atmospheric Environment. 10:925-930.

Le Blanc, F. y Rao, D. 1973. Evaluation of the pollution and drough hypotheses in relation to lichens and bryophytes in urban environmentas. Bryologist. 76: 1-19.

León, Y. 2005. Nuevos registros de musgos (Bryophyta) para el estado Mérida y Venezuela. Plántula.3:149-152.

Matteri, C. 2010. Briofitas. Disponible en: http://www.cricyt.edu.ar/encliclopedia/términos/Briofitas.htm [02 de mayo de 2011].

Mendoza, J. 2018. Evaluación de la calidad del aire empleando líquenes como indicadores en la Ciudad de Chachapoyas, Amazonas, 2017. Tesis para obtener el título profesional de Ingeniera Ambiental. Universidad Nacional Toribio Rodríguez de Mendoza de Amazonas. Perú.

Merwin, M., y Nadkarni, N. 2002. 100 years of tropical bryophyte and lichen ecology: a bibliographic guide to the literatura from 1901-2000. Tropical Bryology. 21:47-67.

Morales, T. 2009. Musgos (Bryophyta) del Parque Nacional Ávila, sectores Cerro El Ávila-Lagunazo, Venezuela. Caldasia. 31:251-267.

Morales, T., Moreno, E y García, M. 2008. Briofitas del área recreativa del Jardín Botánico de Caracas. Ernstia. $18: 37-58$.

Moreno, E. 1992. Aproximación al conocimiento de las briofitas de Venezuela. Tropical Bryology. 6:147-156. 
Munir, S., Shaheen, N., y Nazir, R. 2012. Assessment of the trace elements level in urban atmospheric particulate matter and source apportionment in Islamabad, Pakistan. Atmospheric Pollution Research. 3: 39-45.

Noriega, P., Medici, A., Murillo, A., Bedón, J., Haro, F y Galecio, G. 2008. La Granja. 8:17-24.

Nuñez, E. 2009. Red Autonómica de Biomonitorización de la Contaminación por metales pesados. Informe Final. Disponible: http://www.larioja.org/upload/documents/545918_2009InformeFinal.pdf [10 de mayo de 2010].

Pérez, O. 1984. Estudio del contenido de metales pesados en briófitos del área metropolitana de Barcelona. Anaqueles de Biología. 2:347-352

Pesch, R., y Schroeder, W. 2006. Mosses as bioindicators for metal accumulation: Statisticak aggregation of measurement data to exposure indices. Ecological Indicators. 6(1):137-152.

Pursell, R. 1973. Un Censo de los Musgos de Venezuela. Bryologist, 76(4):473-500.

Ramírez C., y Crusco De DALl'AGLiO, R. 1979. Musgos del Parque Nacional Henri Pittier, estado Aragua, Venezuela. Agronomía Tropical. 10(1-4):403-417.

Rühling, A y Tyler, G. 2001. Changes Atmosfheric Deposition Rates of heavy metal in Sweden a Summary of Nationwide Swedish Surveys en 1968/70-1995. Water, Air y Soil Pollution Focus. 1(3-4):311-323.

Stanković J., Sabovljević, D.,y Sabovljević, M. S2018. Bryophytes and heavy metals: a review. Acta Botanica Croatica. DOI: 10.2478/botcro-2018-0014.

Steinnes, E., Johansen, O., Royset, O. y Odegard, M. 1993. Comparison of different multielement techniques for analysis of mosses used as biomonitors, Environmental monitoring and assessment, 25:87-97.

Taoda, H. 1973. Bry-meter, an instrument for measuring the phytotoxic air pollution. Hikobia. 6:224-228.

Tiller, K.G., 1989. Heavy metals in soils and their environmental significance. Advances in soil Science. 9:113-141.

Tyler, G. 1990. Bryophytes and heavy metals. A literature review. Botanical Journal of the Linnean Society. 104, (13): 231-253. 